\title{
Abuelas, abuelos y padres varones en el cuidado de la infancia*
}

\author{
Gerardo MEIL \\ Universidad Autónoma de Madrid \\ Departamento de Sociología \\ gerardo.meil@uam.es \\ Jesús ROGERO-GARCÍA \\ Universidad Autónoma de Madrid \\ Departamento de Sociología \\ jesus.rogero@uam.es
}

Recibido: 17-04-2013

Aceptado: 18-07-2013

\section{RESUMEN}

Los padres varones han aumentado su participación en el cuidado de los niños en los últimos años. Este trabajo tiene como objetivo conocer si esta creciente implicación de los padres varones sustituye al cuidado de los abuelos o si, por el contrario, existe una relación de complementariedad. Se analiza una muestra procedente de la Encuesta sobre el uso de los permisos parentales compuesta por 1.125 personas con hijos menores de 13 años que viven en pareja y en la que ambos están empleados. Los resultados indican que el cuidado de padres varones y de abuelos tiende a sustituirse y no tanto a complementarse. El análisis también muestra que una parte significativa de parejas de doble ingreso no cuentan con cuidado de abuelos de forma habitual, y que el cuidado de los abuelos paternos no sustituye al de los abuelos maternos -y viceversa-, sino que mantienen una relación de complementariedad.

Palabras clave: abuelos, padres, conciliación, cuidado de niños, masculinidades, división del trabajo doméstico.

\section{The Role of Spanish Grandparents and Fathers in Childcare in Spain}

\begin{abstract}
During the last years, fathers have become more involved on childcare in Spain. The aim of this article is to know whether this increasing involvement of the fathers replaces the care provided by the grandparents or, on the contrary, there is a complementary relationship. We used a sample from the Survey of Parental Leaves Use in Spain, which is composed by 1,125 dual earner respondents with at least one child under 13
\end{abstract}

* Este trabajo forma parte del proyecto de investigación "El uso social de los permisos parentales en España" financiado por la Dirección General de Investigación Científica, Ministerio de Ciencia e Innovación, ref. CSO2009-11328, a quienes los autores agradecen el apoyo recibido. 
years old. Our results show that childcare carried out by fathers and grandparents tend to be substitutive, and not complementary. Our analysis also reveals that a significant part of the dual earner couples does not receive grandparents' support on childcare on a daily basis. The childcare provided by the maternal grandparents does not substitute the childcare carried out by the parental grandparents, but they complement each other.

Keywords: grandparents, fathers, work/family balance, childcare, masculinities, domestic labor division.

\section{REFERENCIA NORMALIZADA}

Meil, G. y Rogero-García, J. (2014). "Abuelas, abuelos y padres varones en el cuidado de la infancia". Cuadernos de Relaciones Laborales, Vol. 32, núm. 1, p. 49-67.

SUMARIO: Introducción e hipótesis de partida. 1. Revisión bibliográfica. 2. Metodología. 3. Resultados. 3.1. Frecuencia del cuidado de niños por los abuelos. 3.2. Cuidado de los abuelos y división del trabajo doméstico. 4. Conclusiones. 5. Bibliografía.

\section{Introducción e hipótesis de partida}

Es un lugar común destacar que los abuelos son un recurso clave en la conciliación de la vida familiar y laboral cuando las madres trabajan. En la literatura sociológica, múltiples estudios destacan que la implicación de los abuelos en el cuidado de los niños es una experiencia muy generalizada en la tercera edad, denunciándose con frecuencia su excesiva implicación. Este aspecto ha sido explorado específicamente en las distintas encuestas que el Centro de Investigaciones Sociológicas (CIS) o el Instituto de Mayores y Servicios Sociales (IMSERSO) han realizado sobre la tercera edad. Según estas encuestas, una proporción cada vez mayor de abuelos ha participado de alguna manera en el cuidado de nietos: mientras que en 1993, un 15\% de los abuelos varones y un $14 \%$ de las abuelas de 65 o más años indicaron que cuidaban de nietos en el momento de la entrevista, en 2006 los porcentajes habían subido hasta el $26 \%$ y $23 \%$ respectivamente (Meil, 2011). En la encuesta realizada por el IMSERSO en 2010, los porcentajes habían aumentado nuevamente alcanzando el $37 \%$ y $33 \%$ respectivamente (IMSERSO, 2010). En conjunto, por tanto, en el plazo de dos décadas, el porcentaje de abuelos de 65 o más años que afirman cuidar niños se habría más que duplicado (concretamente, un incremento del 133\%), aumentando 22 puntos porcentuales en el caso de los abuelos varones y 19 en el de las abuelas. No sólo ha aumentado la proporción de abuelos implicados, sino también la frecuencia con que lo hacen, pues la proporción de abuelos (ambos sexos) que señalan que cuidan a diario ha pasado del $11 \%$ del total de abuelos de 65 o más años en 2006 al $17 \%$ en 2010 . De forma paralela a este proceso, en las últimas décadas se ha producido un incremento en la participación de los hombres en el trabajo doméstico y, particularmente, en el cuidado de los niños (Meil, 2006; García Román y Ajenjo Cosp, 2012).

En este artículo queremos analizar si el cambio en los roles de género de los hombres hacia una mayor implicación en el cuidado de sus hijos se traduce en una menor implicación de los abuelos. Es decir, el objetivo es comprobar si existe una 
relación de sustitución entre el cuidado de los hombres y el cuidado de los abuelos o si, por el contrario, se produce un efecto de complementariedad. Expresado en otros términos, pretendemos conocer si el cambio hacia una familia más igualitaria va acompañado de una menor dependencia de las redes de solidaridad intergeneracional. Se trata de conocer si el modelo de nueva familia extensa del que habla Tobío (2012) es únicamente una fase transitoria o si, por el contrario, la ayuda intergeneracional no responde al reparto del trabajo doméstico en la pareja y a la falta de otros recursos para conciliar vida laboral y familiar, sino que es expresión de un modelo de cuidado caracterizado por el mantenimiento de relaciones intergeneracionales intensas y permanentes.

El artículo se organiza del siguiente modo: el siguiente epígrafe se destina a realizar una revisión bibliográfica y de datos secundarios sobre la implicación de los abuelos y de los padres varones en el cuidado de los niños y los factores de los que dependen; esta revisión nos sirve para fundamentar la hipótesis de partida. En el epígrafe metodológico se presenta la "Encuesta sobre el uso de los permisos parentales en España 2012" en la que se basa este trabajo, la operacionalización de la hipótesis inicial y de las variables utilizadas, así como el modelo de análisis estadístico que se desarrolla. En la parte de los resultados se analizará, en primer lugar, el alcance y características de la colaboración de la red familiar en el cuidado de los niños, para a continuación discutir el impacto de la redefinición de los roles de género en el seno de la familia sobre las ayudas intergeneracionales en el cuidado de niños. Finalmente se presentarán las conclusiones del trabajo.

\section{Revisión bibliográfica}

El aumento de la implicación de los abuelos se ha asociado con la mayor participación de las madres en el trabajo remunerado, los elevados costes del cuidado formal y el limitado alcance de políticas públicas a favor de las familias que, o bien facilitaran escuelas infantiles gratuitas, o permisos parentales remunerados para que los padres pudieran compaginar trabajo y familia (Flaquer, 2001; Tobío, 2008). Esta ausencia de una política familiar realmente destinada a reducir los costes de la tenencia de hijos (Labour Asociados, 2008) habría llevado, según Tobío (2012), a la emergencia de una nueva familia extensa. A ello habría que añadir también las características intrínsecas de este tipo de cuidado: el cuidado de abuelos no suele tener contrapartida económica y es más flexible que otros recursos, como pueden ser las escuelas infantiles, para adaptarse a los requerimientos temporales (cantidad de horas y horario) de la familia que lo demanda. Además, entre los parientes que potencialmente podrían cuidar, los abuelos han sido la alternativa mejor vista por los padres (Pérez Ortiz, 2007). Esta nueva familia en el cuidado de los niños, bien mediante cuidados intensivos en tiempo, sustituyendo a sus hijas mientras trabajan, bien complementando el cuidado realizado por otras personas o instituciones extensa se caracterizaría, según Tobío (2012), sobre todo por la elevada importancia que tienen las abuelas maternas. 
El aumento en el cuidado de abuelos se ha visto acompañado por muchas voces críticas que denuncian una excesiva sobrecarga de la solidaridad familiar, tanto entre los investigadores sociales (Aguinaga, 2004; Pérez Ortiz, 2007; Liga española de la educación y la cultura popular, 2006), llegándose incluso a hablar de "abuelos esclavos" (Guijarro, 2001), como en la percepción de los propios abuelos (Megías y Ballesteros, 2012). Entre éstos, no obstante, predomina la ambigüedad en la evaluación de su propio rol: por un lado, deseo y satisfacción por estar con los nietos y por poder ayudar a los hijos, por otro, sentimiento de ser abuelos "para todo", con una connotación que oscila entre peyorativa y heroica, y hasta cierto punto un sentimiento de ser explotados, sobre todo, entre quienes cuidan a diario. En cualquier caso, los estudios concluyen que, entre los abuelos, hay un discurso dominante sobre el hecho de que los hijos pecan de cierto egoísmo y la necesidad de poner límites a lo que perciben como obligación de ayudarles (Megías y Ballesteros, 2012).

No todas las fuentes, sin embargo, evidencian una implicación tan grande de los abuelos en el cuidado de nietos, sobre todo desde el punto de vista comparativo con otros países. Los datos proporcionados por la encuesta SHARE (The Survey on Health and Retirement in Europe) evidencian, para sorpresa de quienes la han analizado, un limitado alcance de la participación de los abuelos en el cuidado de los niños en el caso español, menor que el registrado en los países con Estados de bienestar más desarrollados. Según la encuesta realizada en 2003 a una muestra representativa de personas de 50 o más años, el porcentaje de abuelos que señalan haber cuidado a sus nietos al menos en alguna ocasión (cuando no conviven con ellos) en el año previo a la encuesta es en España del $40 \%$ para los abuelos varones y del $52 \%$ para las abuelas, que junto con Italia y Suiza son los porcentajes más bajos que se han registrado en los diez países investigados. En el extremo opuesto se encuentra Dinamarca, donde un 59\% de los abuelos y un $72 \%$ de las abuelas han cuidado alguna vez de sus nietos. La media de los diez países incluidos en el estudio se situó en 49\% y 57\%, respectivamente (Hank y Buber, 2007). Ahora bien, si en lugar de considerar a quienes han cuidado en alguna ocasión, se consideran quienes lo hacen de forma regular, "casi semanalmente o con más frecuencia", España se encuentra entre los países en los que existe una mayor proporción de abuelos que cuidan de sus nietos con esta periodicidad (27\% de abuelos varones y $35 \%$ de abuelas, frente a una media de los diez países considerados del $25 \%$ y $32 \%$ respectivamente), aunque esta proporción es menor que en otros países del sur de Europa como Italia y Grecia ( $27 \%$ de abuelos varones y $35 \%$ de abuelas, frente a una media de los diez países considerados del $25 \%$ y $32 \%$ respectivamente). La pauta de cuidado en España, como en otros países del sur de Europa, se caracterizaría entonces por darse con menor frecuencia que en los países del centro y norte de Europa, pero cuando se da, lo es con mayor intensidad (Hank y Buber, 2007; Albertini, Kohli y Vogel, 2007). Otras fuentes de datos, como la Encuesta Europea de Calidad de Vida, en la que se utilizan otros indicadores, también apuntan en la misma dirección (Meil, 2011).

Las razones aducidas para explicar un resultado tan sorprendente apuntan, por un lado, a la posibilidad de atribuir distintos significados al término "ayuda en el 
cuidado de niños" en los distintos países, pudiendo ser que en los países del sur solo se considera que se proporciona ayuda cuando se realiza con una mínima regularidad, mientras que en los demás países habría una mayor predisposición a señalarlo incluso cuando la ayuda es muy ocasional. Por otro lado, se ha aducido que la menor implicación de las madres en el trabajo remunerado en los países del sur haría menos necesaria la ayuda intergeneracional (Hank y Buber, 2007). Al respecto, en la mayoría de los países estudiados los estudios muestran que cuanto mayor es la participación de la madre en el mercado de trabajo, mayor es la implicación de los abuelos en el cuidado de los niños (Aasve, Arpino y Goisis, 2012; Bittman, Craig y Folbre, 2004). A pesar de ello, se ha advertido la necesidad de realizar análisis específicos para cada país, dado que no es posible establecer generalizaciones respecto al cuidado de abuelos a nietos sobre la base de las clasificaciones de países según estado del bienestar comúnmente aceptadas, como la elaborada por Esping-Andersen (1990) (Aasve, Arpino y Goisis, 2012). En el contexto español, Marí-Klose (2010) muestra que cuando la madre está empleada, ya sea a tiempo parcial o completo, la probabilidad de que los abuelos cuiden es mayor, aunque su análisis no integra otros factores relevantes, como la edad de los hijos, la distancia entre el hogar de abuelos y nietos o el uso de otros recursos de cuidado. Para EEUU, se ha observado que las horas de trabajo remunerado de la madre no solo influyen en el tiempo de cuidado de abuelos, sino también en el tipo de actividades en las que se traduce (Vandell et al., 2003).

La incorporación de las mujeres en general y de las madres en particular al trabajo remunerado en España ha aumentado, sin embargo, mucho en las últimas décadas (Moreno, 2007). Según el informe sobre igualdad de género en la Unión Europea (Eurostat, 2008), España es el país donde más aumentó la tasa de empleo de las mujeres en edad de trabajar entre 2000 y 2006, principalmente porque era de las más bajas de la UE, y todavía en la actualidad es menor que en la mayoría de países de la Unión Europea (lo era incluso poco antes de la irrupción de la crisis económica), aunque las diferencias no son muy elevadas. Según evidencian las estadísticas de la Encuesta de Población Activa del INE, la pauta dominante hasta la primera mitad de la década de los años 80 era la salida del mercado de trabajo con la entrada en la maternidad, para a partir del crecimiento del empleo registrado durante la segunda mitad de dicha década, aumentar de forma continuada la incorporación de las mujeres de todas las edades (hasta los 60 años), independientemente de su situación familiar. La tasa de empleo de las mujeres de 25 a 54 años, que abarca todo el período de crianza y atención de los hijos, se mantuvo baja y básicamente constante desde 1976 hasta 1985 (alrededor del 28\%), para aumentar de forma sistemática y continuada a partir de entonces, incluso durante la crisis de empleo registrada en los noventa. La tasa de empleo de este grupo de edad aumentó del $29,4 \%$ de media trimestral en 1985 al 61,4\% en 2012 , con un máximo del $65,9 \%$ en 2008. Ello significa que, aproximadamente, en 6 de cada 10 familias con menores la madre trabaja. Asimismo, según la encuesta en la que se basa este trabajo y que será presentada en el apartado metodológico, en el 
54\% de las parejas con hijos menores de 13 años ambos padres tienen un empleo a comienzos de 2012.

Las diferencias entre géneros en relación con el mercado de trabajo se acentúan entre quienes tienen hijos: los progenitores varones tienen más probabilidades de estar empleados que el resto y dedican más horas al empleo y ocupan tiempos fuera de la jornada ordinaria (tarde, noche y fines de semana) con mucha mayor frecuencia que las madres empleadas (Lewis et al., 2008). La brecha de género en relación con el cuidado de los niños se reduce cuando la mujer está empleada (Craig, 2007), de modo que es posible vincular la mayor participación de las madres en el mercado de trabajo con las nuevas pautas en el cuidado de los padres. Aunque no resulta sencillo identificar las causas de la mayor participación de los hombres en el cuidado de los niños, sí parece evidente que viene acompañada por una redefinición de los roles de género en las esferas familiar y laboral. De este modo, la redefinición del rol social de las mujeres ha ido acompañada de una redefinición del rol familiar de los hombres, que aunque menos profunda y más lenta que en el caso de las mujeres, se ha traducido en una mayor implicación de los hombres en el trabajo doméstico y en cuidado de los niños.

Según la Encuesta de Empleo del Tiempo del INE, el porcentaje de hombres que dedican tiempo a diario al trabajo en el hogar y al cuidado de familiares con los que convive ha aumentado entre 2003 y 2010 en cuatro puntos porcentuales (del $70 \%$ al $74,4 \%$ ) y el tiempo invertido en ello en 24 minutos (de 2 horas y 8 minutos a 2 horas y 32 minutos). La brecha de género sigue siendo, no obstante, grande, pues casi todas las mujeres dedican tiempo a este trabajo $(91,2 \%$ en 2010$)$ y, como media, casi dos horas más (1:57 minutos). Centrando la atención en el grupo de edad de 25 a 44 años, la brecha de género en el porcentaje de personas que cuida niños diariamente ha disminuido de 20 puntos porcentuales en 2003 a 15,6 en 2010, y en lo que se refiere al tiempo invertido en su cuidado, de 47 minutos a 39 minutos. Si en lugar de medir la implicación de los hombres en el trabajo doméstico a través de las estadísticas de uso del tiempo, se utilizan indicadores sobre el reparto entre los cónyuges de las distintas tareas típicas de este tipo de trabajo y del cuidado de niños, también se constata una reducción paulatina de la proporción de familias que están organizadas con arreglo al modelo tradicional y una creciente implicación de los hombres en todas estas tareas. Según dos encuestas realizadas en 1995 y 2003 en Madrid a una muestra representativa de mujeres con hijos menores de 13 años y en las que se pregunta sobre el reparto entre los cónyuges de 13 tareas rutinarias de trabajo doméstico y 12 tareas de cuidado de niños, se observa que en todas las tareas se ha producido una reducción de la proporción de mujeres que señalan que lo hacen ellas exclusivamente y un aumento en casi todas las tareas de la proporción que señala que las hacen a medias con el cónyuge. Agrupando las familias en tradicionales, colaboradoras y cuasi-igualitarias, se observa un descenso del $22 \%$ entre las primeras y del 7\% entre las segundas, al tiempo que un aumento del $186 \%$ de las familias cuasi-igualitarias (Meil, 2006). Otras fuentes, en las que se utilizan otros indicadores también confirman este aumento de la implicación de los hombres, tanto en el trabajo doméstico, como en el cuidado de los niños (García Díez, 2004). 
En esta literatura, si bien la relación entre la situación laboral de la madre y la participación de los abuelos está bien documentada, no ocurre así con los efectos de la situación laboral del padre y de su disposición al cuidado de los niños. La mayoría de estudios internacionales incorporan la situación laboral de la madre (por ejemplo, vid. Borra y Palma, 2009) o la de ambos progenitores sin distinguir su género (por ejemplo, vid. Igel y Szydlik, 2011). En España no hay estudios que relacionen la situación laboral de la madre, del padre, la implicación de este último en el cuidado y la participación de los abuelos en el cuidado. Estas carencias contienen un presupuesto teórico implícito: que el centro articulador del cuidado es la diada madre-hijo/a, en el que la primera establece y coordina los recursos de cuidado de los que dispone el hogar, entre los que destacan el padre y los abuelos.

Respecto a la hipótesis de que una mayor implicación de los padres varones en el cuidado de niños conlleva una menor implicación de los abuelos, aunque no hay estudios cuantitativos que aborden esta cuestión para el caso español, los análisis cualitativos sí ofrecen algunos indicios al respecto. Romero-Balsas et al. (2013) encuentran que los padres que toman permisos laborales más largos para cuidar a sus hijos se muestran reticentes a que los abuelos participen de manera intensa en el cuidado de los niños. Asimismo, desde el punto de vista de los abuelos se ha observado que, en ocasiones, las propias abuelas interpretan que son menos útiles porque los padres varones colaboran más que antes en el cuidado de los hijos (Pérez Ortiz, 2007: 28).

En otros países, la mayoría de los estudios que han abordado el cuidado de abuelos en relación con el reparto del trabajo remunerado y no remunerado en la pareja categorizan a los abuelos junto con otros cuidadores no parentales (personas diferentes al padre o la madre) (Craig, 2007), entre los que suelen distinguirse los de carácter formal e informal (Bittman et al., 2004; Van Dijk y Siegers, 1996). En el contexto italiano, se ha observado que la participación de otros familiares (fundamentalmente abuelos) en el cuidado de los niños se acompaña de una mayor implicación de los padres en tareas rutinarias de cuidado (cambiar pañales, ayudar en el aseo, ayudar a comer, etc.) (Tanturri y Mencarini, 2009). Para Australia, Bittman et al. (2004) encontraron que el uso de recursos informales de cuidado ajenos al hogar se relacionaba con una mayor igualdad en el reparto de cuidado en la pareja. Sin embargo, la vía de la igualdad, en este caso, no derivaba de un aumento significativo en el cuidado de los padres, sino en una sustitución de parte del cuidado materno por otros recursos. Aquí, el mayor cuidado paterno, cuando se producía, se traducía sobre todo en actividades de supervisión y acompañamiento, más que en cuidados físicos o de mayor intensidad. En otro estudio sobre Australia se observó que, entre las madres con empleo, el hecho de recibir ayuda de cuidadores ajenos al hogar no influía en que tuvieran más "tiempo libre sin hijos", sino que generalmente invertían ese tiempo en trabajo remunerado (Craig, 2007). En cambio, para los varones estos apoyos sí revertían en un mayor "tiempo libre sin hijos" (Craig, 2007). Wall, Aboim y Marinho (2007), en un estudio cualitativo sobre padres portugueses, muestran cómo los abuelos facilitan una mayor igualdad en la pareja, tanto en relación con el mercado de trabajo como con el cuidado de los niños. 


\section{Metodología}

Para realizar el análisis propuesto se utiliza la Encuesta sobre el uso de los permisos parentales ${ }^{1}$, cuyo objetivo es conocer los condicionantes familiares, económicos y de género, usos y ventajas e inconvenientes de los permisos para el cuidado de niños y de adultos. Esta encuesta incluye información adicional sobre la participación de diferentes agentes en el cuidado de menores de 13 años. Tiene una muestra de 4.000 personas entre 25 y 60 años, entrevistadas telefónicamente entre enero y marzo de 2012, y es representativa del Estado español excepto Ceuta y Melilla. La muestra analizada está compuesta por 1.125 personas con hijos menores de 13 años con algún abuelo vivo, que viven en pareja y cuando ambos padres tienen un trabajo remunerado en el momento de la entrevista.

La mayoría de fuentes e investigaciones sobre el cuidado de abuelos utilizan como informantes a los propios abuelos (por ejemplo, ver las Encuestas de Personas Mayores del IMSERSO, la Encuesta de Salud, Envejecimiento y Jubilación en Europa (SHARE) o la encuesta a abuelas realizada por Pérez Ortiz (2007)). Este trabajo toma como unidad de observación a uno de los progenitores del/los niño/s que reciben el cuidado. A diferencia de otros estudios, que entrevistan únicamente a madres (por ejemplo, ver Van Dijk y Siegers (1996)), en este caso no se distingue según sexo del progenitor, compensando así el posible sesgo que se deriva del hecho de obtener información de uno de ellos exclusivamente. No obstante, en el análisis multivariable se controla por el sexo del informante.

La variable dependiente utilizada es la frecuencia con la que suelen ayudar los abuelos -maternos o paternos- en el cuidado de los hijos del entrevistado. Las posibilidades de respuesta son diariamente, semanalmente, varias veces al mes, varias veces al año o nunca. En primer lugar, se ha realizado un análisis descriptivo, a través de tablas de contingencia, de la frecuencia con la que los abuelos y otros miembros de la red social participan en el cuidado de menores de 13 años según tipo de vínculo (abuelos maternos o paternos). En segundo lugar, se ha elaborado un modelo de regresión lineal con el objetivo de contrastar la hipótesis principal del trabajo (que existe relación de sustitución entre el cuidado de padres varones y de abuelos). Este modelo selecciona como variable dependiente la frecuencia con la que suelen cuidar los abuelos, que toma valores numéricos de 1 a 5, donde 1 equivale a nunca y 5 a diariamente.

En trabajos precedentes, la implicación de los padres en el cuidado de los niños ha sido medida tanto a través de la frecuencia en la realización de actividades

${ }^{1}$ El cuestionario de esta encuesta fue diseñado por el equipo de investigación del proyecto "El uso social de los permisos parentales en España" (G. Meil, C. García Saínz, M.A. Luque, J. Rogero, D. Muntanyola y P. Romero, Universidad Autónoma de Madrid). El trabajo de campo fue realizado por el Centro de Análisis y Documentación Política y Electoral de Andalucía (CADPEA) (Universidad de Granada). 
(Tanturri y Mencarini, 2009) como mediante el tiempo diario de dedicación (Bittman et al., 2004). En este caso, la variable independiente es el tiempo dedicado al cuidado de niños en un día normal de trabajo (sin contar las horas de sueño). Con el objeto de controlar posibles efectos de otros factores relevantes, se introdujeron dos tipos de variables en el modelo:

(a) Variables relativas al hogar y sus miembros. Edad del hijo más pequeño; número de hijos: "1" = 1, "2" =2 ó "3 o más" = 3; horas de trabajo de la madre: "trabaja menos de 35 horas" $=0$, "trabaja 35 o más horas" $=1$; horas de trabajo del padre: "trabaja menos de 35 horas" = 0 , "trabaja 35 o más horas" $=1$; nivel de ingresos medidos en una escala de seis categorías; sexo del entrevistado: "varón" = 0 , "mujer" = 1. El índice de implicación de los hombres en las tareas domésticas se ha construido sumando las respuestas a la pregunta de quién realiza un conjunto de 5 tareas diferentes $(1=$ siempre la mujer y $5=$ siempre el hombre $)$, tratando los casos en los que lo realiza otra persona como si los hubieran realizado a medias.

(b) Variables relativas al contexto y a otros recursos de cuidado. Los niños comen en el centro educativo: "no" $=0$, "sí" $=1$; distancia en horas a la que viven los abuelos más cercanos; horas semanales de trabajo doméstico remunerado recibido por el hogar: "10h o menos a la semana o no tiene" $=0$, "10h o más a la semana" $=1$.

\section{Resultados}

\subsection{Frecuencia del cuidado de niños por los abuelos}

La ayuda de los abuelos en el cuidado de los niños cuando ambos padres trabajan está muy extendida, pero es menos frecuente de lo que reflejan las encuestas a mayores comentadas en la introducción. Al menos esto es lo que cabe deducir de las respuestas proporcionadas por estas familias respecto a la frecuencia con la que reciben este tipo de ayuda y es coherente con los resultados de otras encuestas (Albertini, Kohli y Vogel, 2007; Hank y Buber, 2007; Meil, 2011). Como puede observarse en la tabla 1, la proporción de familias con hijos menores de 13 años que afirman recibir ayuda de forma diaria es de un tercio (31\%), a lo que hay que añadir un cuarto $(24 \%)$ que señala recibirla de forma semanal. Por tanto, poco más de la mitad (55\%) señala recibir ayuda de forma regular, siendo otra mitad $(45 \%)$ la que la recibe de forma esporádica o no la recibe nunca. 
Tabla 1. Frecuencia con la que las familias con hijos menores de 13 años en las que ambos padres trabajan reciben ayuda en el cuidado de los niños de miembros de su red social. En porcentajes. España 2012

\begin{tabular}{|l|r|r|r|r|}
\hline & $\begin{array}{c}\text { Abuelos } \\
\text { maternos }\end{array}$ & \multicolumn{1}{c|}{$\begin{array}{c}\text { Abuelos } \\
\text { paternos }\end{array}$} & $\begin{array}{c}\text { Algún } \\
\text { abuelo }\end{array}$ & $\begin{array}{c}\text { Otros miembros } \\
\text { de la red* }\end{array}$ \\
\hline Diariamente & $25 \%$ & $14 \%$ & $31 \%$ & $4 \%$ \\
Semanalmente & $22 \%$ & $21 \%$ & $24 \%$ & $7 \%$ \\
Varias veces al mes & $19 \%$ & $18 \%$ & $17 \%$ & $14 \%$ \\
Varias veces al año & $16 \%$ & $19 \%$ & $13 \%$ & $17 \%$ \\
Nunca & $18 \%$ & $29 \%$ & $15 \%$ & $58 \%$ \\
Total & $100 \%$ & $100 \%$ & $100 \%$ & $100 \%$ \\
\hline Nro. de casos & 1.098 & 1.071 & 1.125 & 1.168 \\
\hline
\end{tabular}

* Hermanas y otros familiares, amigos o vecinos.

Fuente: Encuesta sobre el uso de los permisos parentales, 2012. Universidad Autónoma de Madrid.

El cuidado de los abuelos maternos es más frecuente que el de los paternos, como se ha evidenciado en otros estudios (Attias-Donfut, 1995; Pérez Ortiz, 2007; Tobío, 2010, 2012), pero ello no significa que la ayuda de los abuelos paternos sea esporádica o inexistente. El porcentaje de entrevistados que señala recibir esta ayuda a diario es del $14 \%$, a lo que se añade un $21 \%$ que la recibe semanalmente, lo que implica que algo más de una de cada tres familias recibe ayuda de los abuelos paternos cuando ambos padres trabajan. No obstante, hay un sesgo de género a la hora de valorar la implicación de unos abuelos y otros, de forma que las mujeres tienden a señalar que reciben ayuda con menor frecuencia de los abuelos paternos que los hombres, aunque las diferencias no resultan estadísticamente significativas a los niveles convencionales de significación. En el caso de la ayuda recibida de los abuelos maternos no hay diferencias apreciables en la valoración que hacen hombres y mujeres.

Por otra parte, la ayuda de los abuelos paternos no es sustitutiva de la recibida de los abuelos maternos, es decir, los primeros no ayudan cuando los segundos no lo hacen, sino que se complementan; el coeficiente de correlación de Pearson de la ayuda recibida por ambas vías es positivo y además relativamente alto al cifrarse en 0,38 . Por tanto, en términos generales y al margen de lo que pueda suceder en determinados casos, cuanto más frecuente es la ayuda recibida de los abuelos maternos más frecuente es también la ayuda recibida de los abuelos paternos y viceversa. La proporción de familias que señalan recibir ayuda diaria por ambos lados se eleva al 7\% (de las familias que tienen abuelos vivos por ambos lados), mientras que la proporción de quienes señalan no recibirla casi nunca (nunca o solo varias veces al año) por ambas vías es del $28 \%$. El que se reciba ayuda únicamente por parte de una línea de filiación no es la situación más habitual, pero no es infrecuente que suceda, pues se da en un $29 \%$ de las familias entrevistadas en las que hay abuelos por ambas partes. 
La ayuda familiar en el cuidado de niños procede fundamentalmente de los abuelos, pero éstos no son los únicos que proporcionan ayuda en este sentido, como se ha evidenciado también en otros estudios (Meil, 2011). Así, una de cada cuatro familias $(25 \%)$ señala recibir ayuda al menos varias veces al mes de otros miembros de su red social (otros familiares, amigos o vecinos) (tabla 1). En este caso, tampoco hay efectos de sustitución con la ayuda recibida de los abuelos, pues el signo del coeficiente de correlación es también positivo, aunque no muy elevado al no ser tan frecuente este apoyo $(0,15$ con la variable frecuencia de cuidado por parte de alguno de los abuelos).

Estos datos podrían, no obstante, estar sobrevalorando la ayuda recibida, al preguntar por separado por la frecuencia de ayuda recibida por unos abuelos y otros, así como por la recibida de otras personas. Un estudio anterior de Meil (2011) comprobó esta circunstancia al preguntar por el contacto que se mantenía con distintos miembros de la red social. Preguntados específicamente por la frecuencia de contacto con padres y con cada uno de los hermanos e hijos emancipados, la frecuencia de contactos era significativamente superior comparada con la pregunta sobre con qué personas se había mantenido contacto en el fin de semana precedente a la entrevista. En este sentido, un indicador más preciso habría sido preguntar por la frecuencia con que se recibió ayuda en la semana previa a la entrevista.

Tabla 2. Frecuencia con la que las familias con hijos menores de 13 años en las que ambos padres trabajan reciben ayuda en el cuidado de los niños de alguno de los abuelos, según la edad del hijo menor. España 2012

\begin{tabular}{|l|r|r|r|r|r|}
\hline & 0 a 2 años & 3 a 5 años & 6 a 8 años & 9 a 12 años & Total \\
\hline Diariamente & $39 \%$ & $30 \%$ & $26 \%$ & $22 \%$ & $31 \%$ \\
Semanalmente & $27 \%$ & $27 \%$ & $21 \%$ & $18 \%$ & $24 \%$ \\
Varias veces al mes & $17 \%$ & $22 \%$ & $18 \%$ & $14 \%$ & $17 \%$ \\
Varias veces al año & $9 \%$ & $11 \%$ & $16 \%$ & $21 \%$ & $13 \%$ \\
Nunca & $9 \%$ & $11 \%$ & $19 \%$ & $25 \%$ & $15 \%$ \\
Total & $100 \%$ & $100 \%$ & $100 \%$ & $100 \%$ & $100 \%$ \\
\hline Nro. de casos & 424 & 257 & 195 & 247 & 1.123 \\
\hline
\end{tabular}

Fuente: Encuesta sobre el uso de los permisos parentales, 2012. Universidad Autónoma de Madrid.

La ayuda diaria de los abuelos en el cuidado de los niños se da fundamentalmente mientras éstos son muy pequeños, particularmente en el tramo de 0 a 2 años (39\%) y en menor medida en el tramo de 3 a 5 años (30\%). A estas edades es poco frecuente que no se dé nunca o sólo muy esporádicamente y está relacionada, sobre todo aunque no exclusivamente, con la distancia a la que viven los abuelos. A medida que los niños se hacen mayores, la ayuda se vuelve más esporádica, aunque la proporción de familias con hijos de 9 a 12 años que recibe ayuda diaria es todavía relativamente elevada $(22 \%)$, siendo casi mayoría quienes 
no reciben ayuda casi nunca (46\%). La ayuda por parte de otros miembros de la red social está menos estrechamente relacionada con la fase del ciclo familiar, pues aunque es algo más frecuente en el tramo 0 a 2 años, se reparte más homogéneamente en el resto de edades, como lo evidencia el bajo valor del coeficiente de correlación de Pearson entre edad del hijo menor y frecuencia de cuidado ( 0,07 frente a 0,25 en el caso del cuidado de los abuelos).

En conjunto, aunque la ayuda que proporcionan los abuelos en el cuidado de los niños está muy extendida, la ayuda diaria de los abuelos no es la solución más frecuente para conciliar vida familiar y laboral cuando ambos padres trabajan, y ello es así en todas las fases del ciclo familiar, pues incluso en el tramo de edad de 0 a 2 años, casi dos de cada tres familias no reciben esta ayuda de forma diaria (61\%).

\subsection{Cuidado de los abuelos y división del trabajo doméstico}

Para analizar si el proceso de redefinición de los roles de género en el seno de la familia tiene algún impacto en la frecuencia con la que se recibe ayuda de los abuelos, controlando por la influencia de otros factores relevantes, se ha realizado un análisis de regresión lineal, como se ha explicado en el apartado metodológico. Los resultados de este análisis, recogidos en la tabla 3, evidencian que el mayor reparto del trabajo doméstico en la pareja no necesariamente conlleva recurrir con menor frecuencia a los abuelos cuando ambos padres trabajan. La implicación de los abuelos se hace menos frecuente -como refleja el signo negativo del coeficiente beta correspondiente a esta variable $(\beta=-0,073)$ - únicamente si se traduce en una mayor dedicación de tiempo a los niños por parte de los hombres, lo que refleja una mayor implicación en su cuidado y atención. La mayor dedicación a las tareas del hogar por parte de los varones, por el contrario, no condiciona en absoluto la implicación de los abuelos en el cuidado de los niños, a pesar de que está ampliamente demostrado que la implicación de los hombres en el cuidado de los niños es mayor que en las tareas domésticas (Coltrane, 1996; Meil, 2006; Alberdi y Escario, 2007) y de que hay una fuerte correlación entre implicación en las tareas domésticas e implicación en el cuidado de los niños. Las posibles razones para estos resultados divergentes pueden encontrarse, por un lado, en que la implicación de los abuelos suele centrarse exclusivamente en el cuidado de niños y no en la realización de tareas domésticas, aunque no es infrecuente que se reciba ayuda también en este ámbito (Meil, 2011). Por otro lado, hay pautas de redefinición de la división del trabajo doméstico en las que la implicación del hombre se da sobre todo durante el fin de semana o a la noche y se centra más en tareas domésticas que en el cuidado de niños, debido a jornadas laborales muy prolongadas a las que eventualmente se unen tiempos de desplazamientos entre hogar y trabajo elevados o cuando existe una elevada movilidad geográfica. 
Tabla 3. Modelo de regresión lineal sobre la frecuencia con la que las familias con hijos menores de 13 años y cuando ambos padres trabajan reciben ayuda en el cuidado de los niños de alguno de los abuelos*

\begin{tabular}{|c|c|c|c|c|c|}
\hline & \multicolumn{2}{|c|}{$\begin{array}{l}\text { Coeficientes no } \\
\text { estandarizados }\end{array}$} & \multirow{2}{*}{$\begin{array}{c}\text { Coeficientes } \\
\text { tipificados } \\
\text { Beta } \\
\end{array}$} & \multirow[t]{2}{*}{$\mathrm{t}$} & \multirow[t]{2}{*}{ Sig. } \\
\hline & $\mathrm{B}$ & Error típ. & & & \\
\hline (Constante) & 4,665 & 0,357 & & 13,068 & 0,000 \\
\hline Edad del hijo menor & $-0,098$ & 0,011 & $-0,266$ & $-8,607$ & 0,000 \\
\hline Número de hijos & $-0,204$ & 0,068 & $-0,093$ & $-3,012$ & 0,003 \\
\hline Hombre trabaja 35 o más horas & 0,053 & 0,213 & 0,007 & 0,250 & 0,803 \\
\hline Mujer trabaja 35 o más horas & 0,165 & 0,093 & 0,054 & 1,774 & 0,076 \\
\hline $\begin{array}{l}\text { Índice implicación hombres en tareas } \\
\text { domésticas }\end{array}$ & 0,009 & 0,016 & 0,019 & 0,557 & 0,578 \\
\hline $\begin{array}{l}\text { Tiempo dedicado al cuidado de niños } \\
\text { por hombres }\end{array}$ & $-0,047$ & 0,021 & $-0,073$ & $-2,288$ & 0,022 \\
\hline Los niños comen en el centro educativo & $-0,276$ & 0,087 & $-0,099$ & $-3,189$ & 0,001 \\
\hline $\begin{array}{l}\text { Distancia en horas a la que viven los } \\
\text { abuelos más cercanos }\end{array}$ & $-0,055$ & 0,014 & $-0,113$ & $-3,782$ & 0,000 \\
\hline $\begin{array}{l}\text { Tiene empleada hogar } 10 \mathrm{~h} \text { o } \\
\text { más/semana }\end{array}$ & $-0,599$ & 0,135 & $-0,142$ & $-4,456$ & 0,000 \\
\hline Nivel de ingresos & $-0,045$ & 0,032 & $-0,046$ & $-1,419$ & 0,156 \\
\hline Sexo: mujer & $-0,017$ & 0,089 & $-0,006$ & $-0,192$ & 0,848 \\
\hline
\end{tabular}

*Categorías utilizadas en la variable frecuencia del cuidado de abuelos: $1=$ nunca, $2=$ varias veces al año, $3=$ varias veces al mes, $4=$ semanalmente, $5=$ diariamente.

$\mathrm{N}=990, \mathrm{R} 2=0,137$.

En este sentido hay un efecto de sustitución en el cuidado de los niños entre abuelos y padres varones, que, por otro lado, también se produce con otros recursos de conciliación como son la contratación de una persona para ayudar en el trabajo doméstico y el que los niños coman en el centro escolar al que asisten. Cuando los padres varones dedican más horas al cuidado de niños, cuando éstos comen en el centro escolar o guardería y cuando los padres han contratado a una empleada/o de hogar, menor es la implicación de los abuelos en el cuidado de los niños, como se deduce de los niveles de significación de los correspondientes coeficientes del ajuste lineal recogidos en la tabla 3. El efecto sustitución padres varones por abuelos es, no obstante, menor que en los demás casos, como lo evidencia el menor valor del coeficiente tipificado beta del ajuste lineal $(\beta=-0,073$ frente a $\beta=-0,099$ para la variable comer en el colegio y $\beta=-0,113$ para la variable empleada/o de hogar durante al menos 10 horas semanales). La razón para este limitado alcance del efecto sustitución de cuidado por padres varones y por abuelos se debe probablemente a la compatibilidad de horarios entre trabajo y familia, pues los abuelos intervienen típicamente cuando los padres no pueden asumir el cuidado. La tenencia de una persona a la que se paga por el cuidado se adapta lógicamente a las 
necesidades y limitaciones de tiempo de las familias, por lo que es normal que el efecto sustitución con el cuidado de los abuelos sea mayor. Lo mismo sucede con el hecho de que los niños coman en el colegio, pues una forma típica de ayuda de los abuelos es recoger a los niños del colegio y darles de comer al mediodía mientras los padres trabajan.

De forma algo sorprendente, el tiempo dedicado por el hombre al trabajo remunerado no guarda relación con la frecuencia de ayuda recibida por parte de los abuelos, como cabría suponer dado el efecto sustitución entre cuidado por padres varones y cuidado por abuelos. El estimador de esta relación no es estadísticamente significativo tanto si se mide como variable continua (por tramos de 10 horas), como si se hace contraponiendo tiempo completo a tiempo parcial, como se ha recogido en el ajuste presentado en la tabla 3. Una posible razón de esta ausencia de relación puede encontrarse en el hecho de que menor tiempo de trabajo no implica necesariamente mayor tiempo de cuidado de los hijos (Craig, 2007) y, por tanto, menor necesidad de ayuda externa, pues los hombres pueden preferir dar otros usos al tiempo que no dedican al trabajo remunerado. Lo relevante, por tanto, no es el tiempo dedicado al trabajo, sino el tiempo que se dedica al cuidado de niños.

En el caso de las mujeres, sin embargo, el tiempo dedicado al trabajo remunerado sí condiciona el grado de ayuda recibida de los abuelos, de forma que cuanto más dedica la mujer al trabajo remunerado, más frecuente es la ayuda recibida. Esta relación, sin embargo, no se evidencia de una forma muy clara y proporcional. Si se introduce el tiempo de trabajo remunerado como variable lineal (medida en tramos de 10 horas), no existe asociación entre ambas variables, lo que significa que a mayor tiempo de trabajo remunerado no se da necesariamente mayor implicación de los abuelos, incluso controlados los efectos de la edad del niño, participación del cónyuge en el cuidado y demás variables consideradas. Únicamente si se distingue entre si tiene trabajo a tiempo completo o tiempo parcial, se evidencia que las mujeres con trabajo a tiempo parcial (menos de 35 horas semanales) recurren en menor medida a los abuelos que las que trabajan a tiempo completo, aunque el nivel de significación del estimador se sitúa algo por encima del nivel convencional de significación comúnmente aceptado de 0,05 (concretamente 0,076 ). Por otra parte, su impacto es muy limitado, más incluso que el del tiempo dedicado por los hombres al cuidado de los niños $(\beta=0,054)$.

Más allá de las circunstancias indicadas, el análisis realizado evidencia también que la distancia a la que viven los abuelos condiciona la frecuencia con la que se recibe la ayuda, de forma que cuanto más cerca viven, más frecuente es la ayuda recibida, un resultado ampliamente documentado en la literatura sobre las relaciones integeneracionales (Baydar y Brooks-Gunn, 1998; Hank y Buber, 2007; Tobío, 2010; Meil, 2011). Por otra parte, y de forma sorprendente, los resultados muestran también que la frecuencia de la ayuda disminuye no sólo con la edad de los niños, sino también con el número, de forma que a mayor número de niños, menor frecuencia de ayuda recibida. Estos datos sugieren que la mayor frecuencia de ayuda no sólo se concentra en los primeros años de vida del nieto, sino también con los primeros nietos. De esta forma, cuando la familia crece, más que aumentar 
la ayuda como consecuencia de las mayores dificultades de conciliación y la mayor carga de trabajo, las generaciones optan por otras soluciones para conciliar vida familiar y laboral, volviéndose más esporádico el apoyo de los abuelos.

\section{Conclusiones}

Dos procesos de ritmo desigual están cambiando las relaciones familiares en los últimos años: la incorporación de las mujeres al mercado de trabajo y la mayor participación de los hombres en la esfera doméstica. En España, el acceso de muchas madres al empleo se ha apoyado en la ayuda de los abuelos en el cuidado de los niños. Este trabajo confirma que las familias en las que ambos padres trabajan reciben frecuentemente la ayuda de los abuelos para cuidar a los niños y que la ayuda recibida de los abuelos paternos no es sustitutiva, sino complementaria a la ayuda de los abuelos maternos. No obstante, la mayoría de las familias no cuenta con este recurso de manera habitual.

Por su parte, la mayor implicación de los varones en el cuidado de los niños podría estar modificando las pautas de organización de las parejas y, por ende, su demanda de ayuda a otras personas. El objetivo principal de este trabajo era analizar hasta qué punto los padres están sustituyendo a los abuelos como agentes facilitadores del acceso de las mujeres al mercado de trabajo. Nuestros resultados sugieren que las familias en las que los hombres están más implicados demandan menos apoyo intergeneracional, de modo que el cuidado de padres varones y de abuelos tiende a sustituirse y no tanto a complementarse. De acuerdo con esto, si la tendencia a una mayor implicación de los padres continúa, es de prever que la ayuda de los abuelos tenga menor demanda por parte de las familias. La implicación de los abuelos disminuye también cuando existen recursos de apoyo remunerados, como pueden ser las empleadas/os de hogar o los centros educativos.

Este trabajo presenta también algunas limitaciones. En primer lugar, la encuesta utilizada carece de información detallada sobre los abuelos que cuidan (sexo, situación laboral, estado civil, etc.), datos que contribuirían a matizar los resultados al considerar factores relevantes identificados por la literatura. En segundo lugar, los datos son transversales. La información longitudinal o pseudo-longitudinal (misma fuente realizada en momentos diferentes con muestras distintas) contribuiría a identificar tendencias en el cuidado de los abuelos con mayor solidez.

A pesar de sus limitaciones, este trabajo aporta conocimiento nuevo y relevante sobre el cuidado de abuelos a niños en España. Se ofrecen datos actualizados sobre su incidencia y, por primera vez, se explora la interrelación entre el cuidado de los padres varones y de los abuelos. Los resultados apuntan a que el mayor protagonismo de los primeros podría desembocar en una pérdida de peso de los últimos en la crianza de los niños. Si así fuera, el camino hacia un cuidado de los abuelos más respetuoso con su calidad de vida pasa por una mayor participación de los hombres en el cuidado de sus hijos, así como por el establecimiento de más recursos para la conciliación de las familias con niños a su cargo. No obstante, 
queda abierta la cuestión de hasta qué punto la implicación de los abuelos constituye un freno a una mayor implicación de los hombres en el cuidado, y de si los padres varones se implican más cuando no disponen del apoyo de los abuelos.

\section{Bibliografía}

Aasve, A., Arpino, B. y Goisis, A. (2012). Grandparenting and mother's labour force participation: A comparative analysis using the generations and gender survey. Demographic Research. Vol. 17 Núm. 3, 53-84.

Aguinaga, J. (2004). El precio de un hijo: Los dilemas de la maternidad en una sociedad desigual. Madrid: Debate.

Alberdi, I., y Escario, P. (2007). Los hombres jóvenes y la paternidad. Bilbao: Fundación BBVA.

Albertini, M., Kohli, M. y Vogel, C. (2007). Intergenerational transfers of time and money in European families: common patterns - different regimes? Journal of European Social Policy. Vol. 17 Núm. 4, 319-334.

Attias-Donfut, C. (ed) (1995). Les solidarités entre générations. Vieillesse, Familles, État. Paris: Nathan.

Baydar, N. y Brooks-Gunn, J. (1998). Profiles of grandmothers who help care for their grandchildren in the United States. Family Relations. Vol. 47 Núm. 4, 385-393.

Bittman, M., Craig, L., y Folbre, N. (2004). Packaging care: What happens when parents utilize non-parental child care. En M. Bittman y N. Folbre (eds.) Family time: The social organization of care. London: Routledge.

Borra, C. y Palma, L. (2009). Child care choices in Spain. Journal of Family and Economic Issues. Vol. 30, 323-338.

Coltrane, S. (1996). Family Man: Fatherhood, housework and gender equity. Newbury Park: Pine Forge Press.

Craig, L. (2007). How Employed Mothers in Australia Find Time for Both Market Work and Childcare. Journal of Family and Economic Issues. Vol. 28, 69-87.

Eurostat (2008). The life of women and men in Europe. Luxemburgo: Eurostat statistical books, disponible en http://epp.eurostat.ec.europa.eu/cache/ITY_OFFPUB/KS-80-07-135/EN/KS-8007-135-EN.PDF 
Flaquer, L. (2001). Las políticas familiares en una perspectiva comparada. Barcelona: Fundación La Caixa.

García Díez, S. (2004). Análisis socioeconómico de la estructura productiva de los hogares. Madrid: Consejo Económico y Social.

García Román, J. y Ajenjo Cosp, M. (2012). Cambios en el uso del tiempo de las parejas. ¿Estamos en el camino hacia una mayor igualdad? Papers de Demografia 375. Centre D’Estudis Demogràfics.

Guijarro, A. (2001). El síndrome de la abuela esclava. Granada: Grupo editorial universitario.

Hank, K y Buber, I. (2007). Grandparents Caring for Their Grandchildren: Findings from the 2004 Survey of Health, Ageing and Retirement in Europe. MEAMannheim Research Institute for the Economics of Ageing. 127-2007.

Igel, C. y Szydlik, M. (2011). Grandchild care and welfare state arrangements in Europe. Journal of European Social Policy. Vol. 21, 210-224.

IMSERSO (2010). Encuesta mayores 2010, microdatos. Disponibles en http://www.imsersomayores.csic.es/estadisticas/index.htm.

Labour Asociados (2008). Hacia un nuevo modelo de protección a las familias en España. Secretaría de Estado de la Seguridad Social, Expediente FIPROS 2007/36, disponible en http://www.segsocial.es/Internet 1/Estadistica/FondodeInvestigacio48073/EstudiosFIPROS/ Tema6Otros/index.htm

Lewis, J, Campbell, M. y Huerta, C. (2008). Patterns of paid and unpaid work in Western Europe: gender, commodification, preferences and the implications for policy. Journal of European Social Policy. Vol. 18, 21-37

Liga española de la educación y la cultura popular (2006). Valoración del tamaño y condiciones de vida de los hogares formados por abuelos pensionistas con nietos a su cargo. Madrid: Ministerio de Trabajo y Asuntos Sociales.

Marí-Klose, P.; Marí-Klose, M; Vaquera, E. y Argeseanu, S. (2010). Infancia y futuro: Nuevas realidades, nuevo retos. Barcelona: Fundación La Caixa.

Megías, I y Ballesteros, J. C. (2011). Abuelos y abuelas... para todo: percepciones en torno a la educación y el cuidado de los nietos. Madrid: Fundación de Ayuda contra la Drogadicción. 
Meil, G. (2006), Continuidad y cambio en la división del trabajo doméstico en la familia urbana en Varios Autores, Libro homenaje a José Cazorla (pp 449-468). Madrid: Centro de Investigaciones Sociológicas.

Meil, G. (2011). Individualización y solidaridad familiar. Barcelona: Fundación La Caixa.

Moreno Mínguez, A. (2007). Modelos familiares y empleo de la mujer en el Estado de bienestar español. Estudios de Progreso Fundación Alternativas, 25/2007.

Nicholson, L. y Davey Zeece, P. (2008). Grandparents in the Lives of Young Children. Redefining Roles and Responsibilities. En M.R. Jalongo (ed.), Enduring Bonds (pp 129-144). Indiana: Springer.

Pérez Ortiz, L. (2007). Las abuelas como recurso de conciliación entre la vida familiar y laboral, Presente y futuro. Madrid: Instituto de la Mujer.

Romero-Balsas, P., Muntanyola-Saura, D. y Rogero-García, J. (2013). DecisionMaking Factors within Paternity and Parental Leaves: Why Spanish Fathers Take Time Off from Work. Gender, Work \& Organization. Doi: 10.1111/gwao.12004.

Tanturri, M.L. y Mencarini, L. (2009). Fathers involvement in daily childcare activities in Italy: does a work-family reconciliation issue exist? CHILD Working Papers 22.

Tobío, C. (2008). Redes sociales, género y política social en España y Francia. Política y Sociedad. Vol. 45 Núm. 2, 87-104

Tobío, C. y Caballero, F. y González, M.E. (2010). Abuelas y abuelos en la red familiar. Sevilla: Instituto de Estadística de Andalucía.

Tobío, C. (2012). Reciprocity and solidarity in intergenerational relationships: Spain, France and Norway in comparative perspective. Papers. Vol. 97 Núm. 4, 849-873.

Van Dijk, L. y Siegers, J.J. (1996). The Division of Child Care Among Mothers, Fathers, and Nonparental Care Providers in Dutch Two-Parent Families. Journal of Marriage and the Family. Vol. 58, 1018-1028

Vandell, D. L., McCartney, K., Owen, M. T., Booth, C. y Clarke-Stewart, A. (2003). Variations in child care by grandparents during the first three years, Journal of Marriage and Family. Vol. 65 Núm. 2, 375-381. 
Wall, K. Aboim, S. y Marinho, S. (2007). Fatherhood, Family and Work in Men's Lives: Negotiating New and Old Masculinities. Researches Sociologiques et Anthropologiques. Vol. 38-2, 105-122

Zamarro, G. (2011). Family Labor Participation and Child Care Decisions. The Role of Grannies. RAND Labor and Population Working Papers WR-833. 